\title{
Advances in transcriptome analysis of human brain aging
}

\author{
Seokjin $\mathrm{Ham}^{1}$ and Seung-Jae V. Lee (1)
}

\begin{abstract}
Aging is associated with gradual deterioration of physiological and biochemical functions, including cognitive decline. Transcriptome profiling of brain samples from individuals of varying ages has identified the whole-transcriptome changes that underlie age-associated cognitive declines. In this review, we discuss transcriptome-based research on human brain aging performed by using microarray and RNA sequencing analyses. Overall, decreased synaptic function and increased immune function are prevalent in most regions of the aged brain. Age-associated gene expression changes are also cell dependent and region dependent and are affected by genotype. In addition, the transcriptome changes that occur during brain aging include different splicing events, intersample heterogeneity, and altered levels of various types of noncoding RNAs. Establishing transcriptome-based hallmarks of human brain aging will improve the understanding of cognitive aging and neurodegenerative diseases and eventually lead to interventions that delay or prevent brain aging.
\end{abstract}

\section{Introduction}

Aging is a nearly universal biological process associated with gradual deterioration in physiological and biochemical functions, including cognitive decline ${ }^{1,2}$. Aging is also a major risk factor for many neurodegenerative diseases, including Alzheimer's disease and Parkinson's disease ${ }^{2,3}$. This close relationship between aging and neurodegenerative diseases suggests the existence of common transcriptional and posttranscriptional gene regulation mechanisms in the brain.

Age-associated cognitive declines usually correlate with corresponding age-related structural changes in the brain, such as neuronal loss and synaptic changes ${ }^{1,4}$. However, the correlation is not universal. For example, changes in gene expression profiles in the brain during aging can precede apparent histopathological degeneration of the brain and appear to underlie age-associated cognitive declines at the molecular level ${ }^{5-8}$. Therefore, transcriptome analysis of brain aging offers a systematic

Correspondence: Seokjin Ham (sjham@kaist.ac.kr) or Seung-

Jae V. Lee (seungjaevlee@kaist.ac.kr)

${ }^{1}$ Department of Biological Sciences, Korea Advanced Institute of Science and

Technology, 291 Daehak-ro, Yuseong-gu, Daejeon 34141, South Korea molecular approach to understand the causes of neurocognitive aging and neurodegenerative diseases.

The transcriptome is defined as "the complete complement of mRNA molecules generated by a cell or population of cells"9,10. The information of cell populations is stored in the genome; therefore, transcriptome profiling is important for interpreting functional genomic elements $^{11}$. The objectives of transcriptomics, or the study of the transcriptome, are to categorize all transcripts, to analyze the variants of expressed genes and to quantify the levels of transcripts under different conditions $^{12}$. The subsequent functional characterization of the genes and transcripts of interest obtained from transcriptome analyses also helps validate their biological relevance.

Transcriptome profiling of brain samples from individuals of varying ages is a useful strategy for studying human brain aging by allowing the detection of overall age-associated transcriptome changes. Although transcriptome profiling alone generates descriptive information, assessment and interpretation of this information allows researchers to effectively design subsequent functional analyses of candidate genes and transcripts ${ }^{11,13}$. 


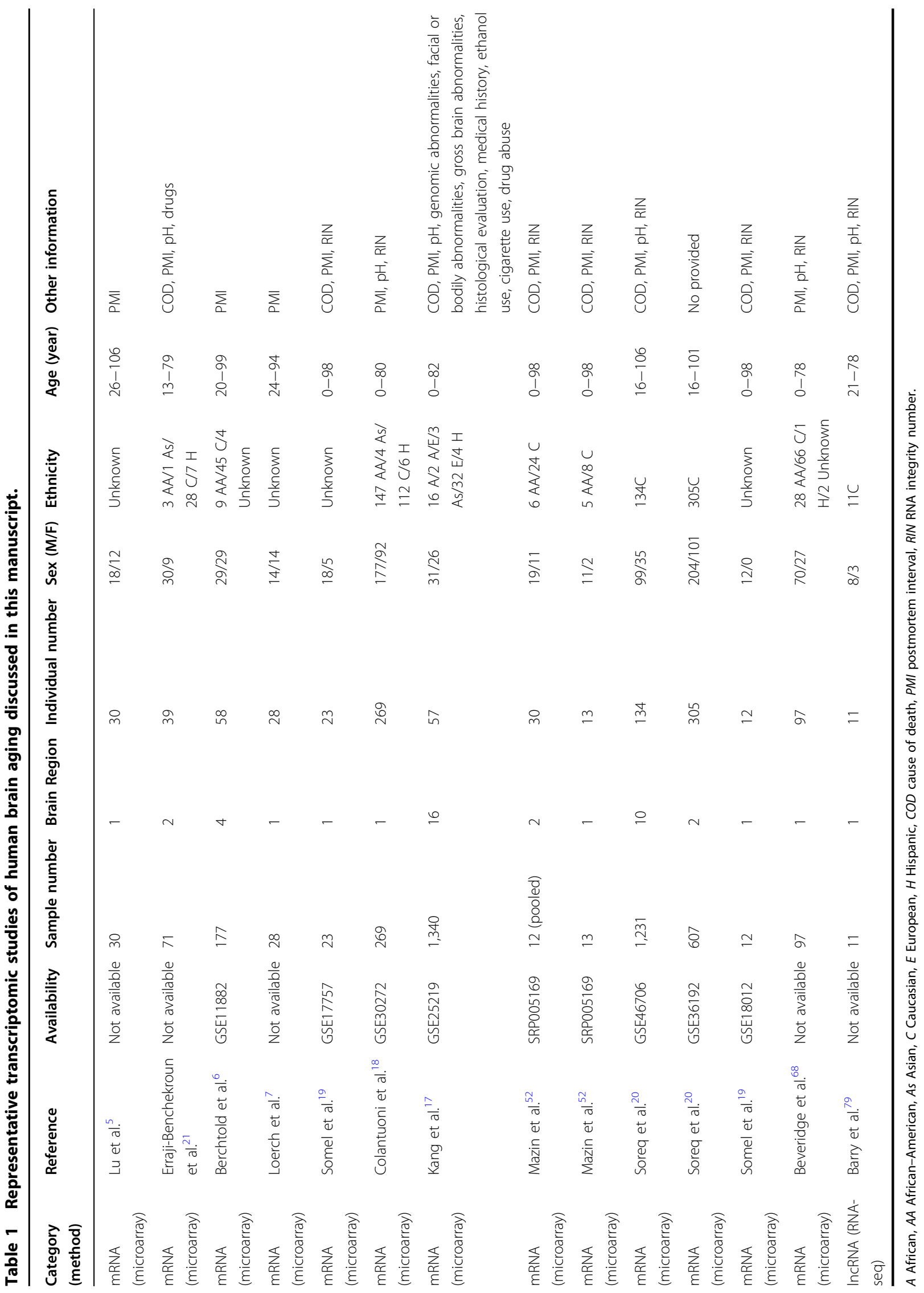




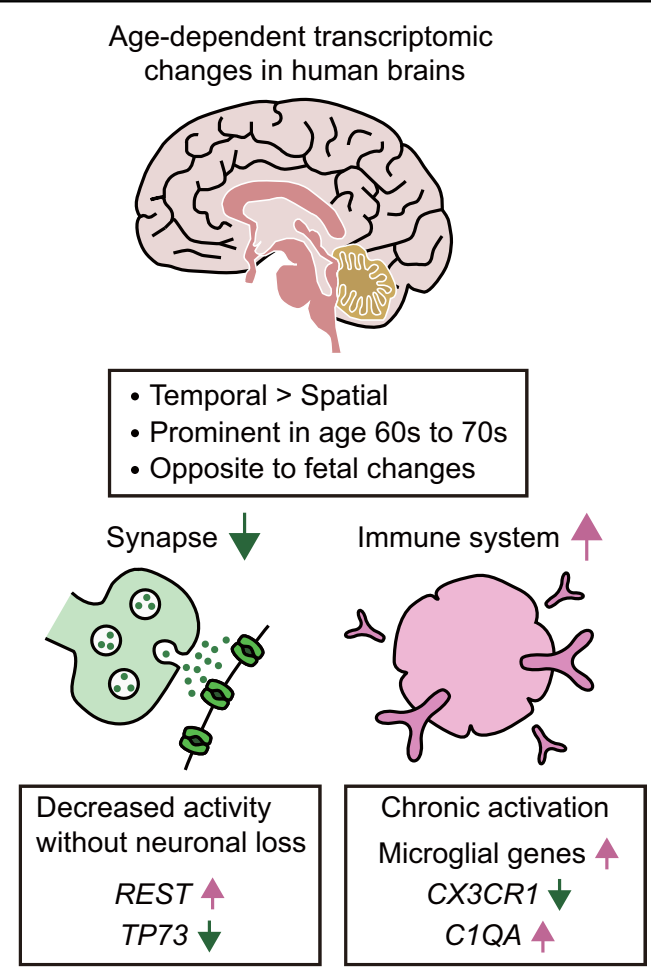

Fig. 1 Global age-dependent transcriptomic changes in human brains. Expression changes are mainly temporal by relative preservation of their spatial identity. Substantial expression changes are detected in the sixth and seventh decades of human life. The directions of gene expression changes during adult aging tend to be opposite those occurring during fetal development. Throughout multiple brain regions, age-dependent transcriptomic changes include decreased synaptic function and increased immunity. It is worth noting that synaptic aging occurs even prior to neuronal loss. Decreased synaptic function underlies increased expression of repressor element-1-silencing transcription factor (REST) and decreased expression of the tumor protein 73 (TP73) gene. Increased immune responses are accompanied by upregulation of microglial genes and complement component 1q A (C1QA) and downregulation of genes encoding immunosuppressive factors, including C-X3-C motif chemokine receptor 1 (CX3CR1).

Because human postmortem tissues are difficult to obtain, progress in transcriptome profiling of human brain aging has been very slow. However, international collaboration for the collection of human brain samples and subsequent generation of various sets of experimental data has paved the way for molecular biological research of human brain aging ${ }^{14-16}$. This collaboration has thus contributed to the comprehensive understanding of aging-related transcriptomic changes in the human brain.

In this article, we review and discuss studies based on transcriptome analyses of human brain aging (Table 1 ). We categorize the subtopics of transcriptome analysis into global gene expression changes, cell type-specific expression changes, gene coexpression networks, expression quantitative trait loci, and alternative splicing.
Decreased synaptic function and increased immune function are prevalent in most analyses, in addition to other features that have been detected in some studies. Increased intersample heterogeneity is also an indispensable factor in the understanding of human brain aging. In addition, we address age-associated expression changes in various types of noncoding RNAs, including microRNAs (miRNAs), long noncoding RNAs (lncRNAs), and circular RNAs (circRNAs). Transcriptome analysis of human brain aging will pave the way for the early diagnosis and prevention of cognitive aging and ageassociated brain diseases.

\section{Age-dependent transcriptome changes in the brain include decreased synaptic function and increased immune function}

One of the most extensive transcriptome analyses on human brain aging with a large sample size and diverse ethnicity provided insights into global changes across the whole brain, as well as in specific regions ${ }^{17}$. Generally, temporal regulation of protein-coding genes is more pronounced than spatial or regional regulation (Fig. 1). The expression of $57.7 \%$ of genes is changed during prenatal development. In addition, $9.1 \%$ of the gene transcripts that are expressed in the neocortex are changed during postnatal development (an age of under 20 years), but only $0.7 \%$ of them are altered during adulthood (an age of over 20 years). These data indicate that the majority of differentially expressed genes display temporal changes during development and that the effects of aging on gene expression changes are relatively minor.

However, research using adult brains has indicated noticeable transcriptional changes during adult aging (Fig. 1). Another comprehensive transcriptome study analyzing the prefrontal cortex of 269 human individuals indicates that reduced rates of gene expression changes in the early postnatal brain are maintained throughout early childhood and through the first four decades of life ${ }^{18}$. Thereafter, substantial gene expression changes across multiple brain regions are detected in the sixth and seventh decades of human life, based on an analysis mostly including people of Caucasian ancestry ${ }^{6,18}$, indicating the existence of a qualitative transition phase (Fig. 1). The majority of the gene expression changes observed during adult aging are similar to those detected during postnatal development, which includes infancy, childhood, and puberty; interestingly, the directions of these changes are opposite those observed during fetal development ${ }^{18,19}$ (Fig. 1). In addition, males display a larger number of gene expression changes across multiple brain regions with aging than females ${ }^{6,17}$. In contrast to this sexual dimorphism, a study that analyzed two large data sets containing over 1800 human samples shows that the expression of genes exhibits similar changes with aging across brain regions ${ }^{20}$. 
Notably, many independent transcriptome analyses have commonly indicated that genes that are upregulated during aging in most brain regions are enriched in inflammatory or immune-responsive genes annotated in the Gene Ontology (GO) database $e^{5,6,20-25}$ (Fig. 1). Although appropriate immune activation is neuroprotective, chronic immune activation appears to increase the vulnerability of the brain to neurocognitive aging and neurodegenerative diseases ${ }^{22,23,26}$. In contrast, multiple studies have repeatedly reported that genes that are downregulated across multiple brain regions during aging are enriched with genes related to synaptic transmission and plasticity ${ }^{5-7,21,24,25,27,28}$ (Fig. 1). These findings suggest that age-associated cognitive decline is driven by synaptic aging without neuronal loss ${ }^{1,5}$. Consistent with these observations, accumulating evidence indicates that dysregulation of intracellular calcium ion homeostasis is linked to age-related cognitive declines ${ }^{29,30}$. Genes that exhibit age-dependent expression changes in brain regions are enriched in other biological processes as well; these processes include neurotransmitter transport, energy metabolism, chaperone response, apoptosis, RNA metabolism, amino acid biosynthesis, DNA repair, mitochondrial function, and reactive oxygen species metabolism ${ }^{5,19,21,27,31-33}$. Many of these processes appear to be neuroprotective.

Recent transcriptomic studies in combination with thorough molecular genetic analysis indicate that repressor element-1-silencing transcription factor (REST), a neuronal repressor that is crucial for brain development, functions as a neuroprotective factor against brain aging $^{34}$. The expression levels of REST are increased in normal aged brains but not in the brains of individuals with mild cognitive impairment or Alzheimer's disease $^{25,34}$. REST downregulates excitatory synaptic transmission and upregulates the longevity-associated transcription factor forkhead box O1 (FOXO1) in the mammalian brain, as well as its homolog DAF-16 in Caenorhabditis elegans to promote longevity ${ }^{25}$. These studies regarding REST suggest the existence of active protective mechanisms in the brain against neurodegeneration and the contribution of reduced excitatory synaptic transmission to longevity (Fig. 1).

Age-dependent gene expression changes in specific cell types have also been detected in diverse brain regions. Multiple papers have commonly reported that neuronspecific genes are predominantly downregulated in all brain regions during aging $7,19,21,33,35$, but these genes display region-specific, age-dependent expression changes by preserving their regional identity ${ }^{20}$. In contrast, gliaspecific genes with age-dependent alterations in their expression lose their regional identity, and their expression is shifted to multiple regions ${ }^{20}$. Microglia act as innate immune cells of the central nervous system ${ }^{36}$.
During aging, microglia-specific genes are generally upregulated in all brain regions, with the exception of the cerebellum $^{20,21,23,26}$ (Fig. 1). In addition, endothelial cellspecific genes are transcriptionally upregulated across multiple brain regions during aging ${ }^{20}$. Oligodendrocytes act as supporting cells for neurons in the central nervous system by forming myelin sheaths around axons ${ }^{37}$. In contrast to the expression level changes in microgliaspecific and endothelial cell-specific genes, the expression levels of oligodendrocyte-specific genes tend to decrease in various brain regions during aging ${ }^{20,35}$. These findings provide evidence supporting the occurrence of global and cell- or region-specific transcriptional changes during human brain aging.

\section{Gene coexpression network analysis supports decreased synaptic function and increased immune function during brain aging}

Aging is regulated by complex interplay among various components; ${ }^{2,3,8}$ therefore, the transcriptomes of aged samples usually exhibit high intersample variation. Gene coexpression network analysis is utilized for detecting modules or clusters of highly correlated genes associated with specific functions and for obtaining key information embedded in heterogeneous transcriptomes ${ }^{38}$ (Fig. 2a). A network analysis of published transcriptomic data obtained from adult hippocampus samples has indicated that a module of genes that display age-dependent upregulation is enriched in myelination ${ }^{39}$. This study also confirmed an immune process-enriched module that correlated positively with age and a synaptic transmissionenriched module that correlated negatively with age ${ }^{39}$.

Although decreased synaptic function has been reproducibly reported in aged prefrontal cortices ${ }^{40}$, the increase in immune function in aged brains has been disputed by one study that analyzed three data sets obtained from the frontal cortices of 381 individuals $^{41}$. Specifically, the expression levels of multiple genes in a microglia-specific module, including microglia surface receptors and Tolllike receptors, decrease in the prefrontal cortex with age $^{41}$. Thus, some functions of microglia appear to decline during brain aging. However, the microglia module also contains genes encoding immunosuppressive factors, including $\mathrm{C}-\mathrm{X} 3-\mathrm{C}$ motif chemokine receptor 1 $\left(\mathrm{CX}_{3} \mathrm{CR} 1\right) ;{ }^{41}$ therefore, the balance between immune activation and suppression is important for appropriate immune responses ${ }^{6,22}$. For instance, the microglia-specific neuroprotective receptor for fractalkine (FKN), $\mathrm{CX}_{3} \mathrm{CR} 1$, suppresses excessive immune responses via microglia activation in mice and rats $^{42,43}$. Signaling through the FKN ligand and its receptor, $\mathrm{CX}_{3} \mathrm{CR} 1$, in microglia is reduced in aged brains ${ }^{22,41,43}$, and this reduction likely contributes to neurotoxic inflammation and results in neurodegeneration (Fig. 1). However, we cannot rule out 
a

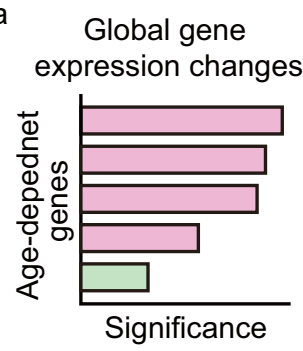

Immune-related Synapse-related

b

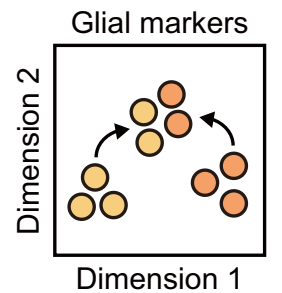

Neuronal markers

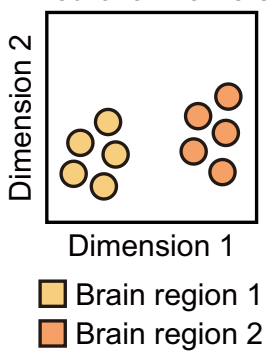

Network analysis of gene co-expression

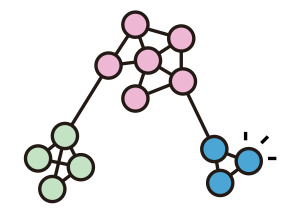

Myelination-related
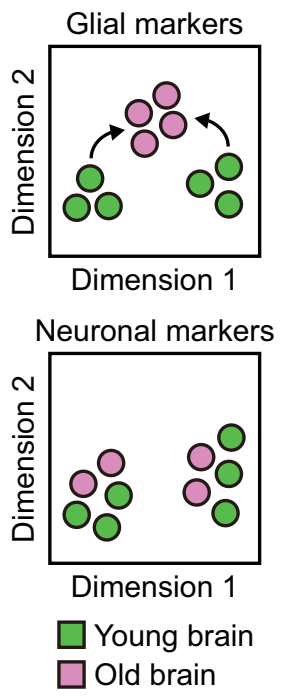

Fig. 2 Targeted analysis of transcriptomic changes during human brain aging. a Age-dependent upregulation of myelination-related genes, which is undetectable in global analysis, is detected via gene coexpression network analysis. Genes that exhibit highly positive correlations among themselves are connected to one another. Different colors represent different groups of genes related to specific functions. b Glia-specific genes lose their regional identity during brain aging, whereas neuron-specific genes preserve their regional identity. Glial markers include genes that are specifically expressed in astrocytes and oligodendrocytes. The dimensions of the transcriptome are reduced for visualization by principal component analysis or multidimensional scaling.

the possibility that age-dependent downregulation of a subset of microglia-specific genes may reflect the complex regulation of these genes, as well as the altered number of microglia during aging. Collectively, these findings based on experimental data from multiple studies show that decreased synaptic function and increased immune function are predominant during human brain aging.

\section{Glia-specific genes lose their regional identity during brain aging}

Examination of the age-dependent changes in the expression of cell-type-specific genes reveals that genes

specific to glia, including astrocytes and oligodendrocytes, exhibit altered regional expression patterns during brain aging $^{20}$ (Fig. 2b). These changes in the regional expression of glia-specific genes is most prominent in the hippocampus in the forebrain and in the substantia nigra in the midbrain, both of which are susceptible to Alzheimer's disease and Parkinson's disease pathogenesis. For example, the expression of astrocyte-specific genes in the hippocampus is similar to that observed in the cortex in young brains; however, the expression of these genes shifts toward that observed in intralobular white matter and the putamen in aged brains. Oligodendrocyte-specific genes exhibit differential expression in the hippocampus and in the substantia nigra in young brains; however, their expression in these structures becomes similar in aged brains. In contrast, neuron-specific genes preserve their regional identity during brain aging (Fig. 2b). Thus, during human brain aging, the expression of genes in specific cell types, including astrocytes and oligodendrocytes, changes dramatically and regional identities are lost.

\section{Analysis of expression quantitative trait loci (eQTLs) reveals that genetic backgrounds affect gene expression in an age-dependent manner}

Quantitative trait locus (QTL) analysis is a method that is used to infer the effects of genetic background on different traits. Expression QTLs (eQTLs) are genomic loci that are highly related to the expression levels of target genes $^{44,45}$. Many eQTLs show consistent effects on the expression levels of their targets independent of age ${ }^{17,18}$. Interestingly, however, several studies using less stringent thresholds or a particular type of cell, such as microglia, have reported that some of these loci display differential effects on the brain at different ages ${ }^{26,32}$. One example is the single-nucleotide polymorphism (SNP) rs55675298 ${ }^{32}$; individuals with the GG allele show an age-dependent increase in the expression of the tumor protein 73 (TP73) gene, which encodes a member of the p53 protein family, whereas individuals with the GT or TT alleles do not exhibit this increase (Fig. 3a). TP73 is critical for normal neuronal development and survival in mice ${ }^{46-48}$; therefore, age-dependent changes in the expression of TP73 potentially affect brain aging and age-related diseases (Fig. 1). Indeed, the allele frequency of TP73 is implicated in human Alzheimer's disease ${ }^{49}$, and haploinsufficiency of TP73 confers susceptibility to Alzheimer's disease in model mice ${ }^{50}$. Similarly, the GG allele of the SNP rs72788737 is associated with age-dependent increases in the expression of complement component 1q A (C1QA), which encodes the $\mathrm{C} 1 \mathrm{q}$ protein responsible for innate immune responses, whereas the GT or TT allele is not ${ }^{32}$. The expression levels of $C 1 Q A$ increase during normal brain aging (Fig. 1), and the C1q protein accumulates in several brain regions, including the hippocampus, 

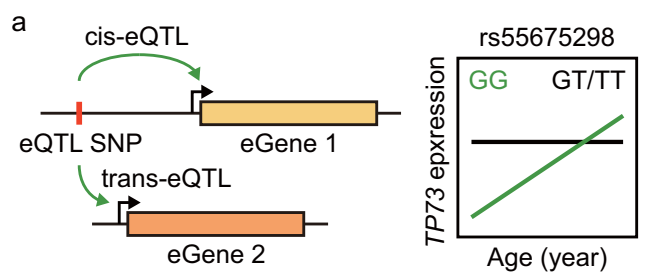

b

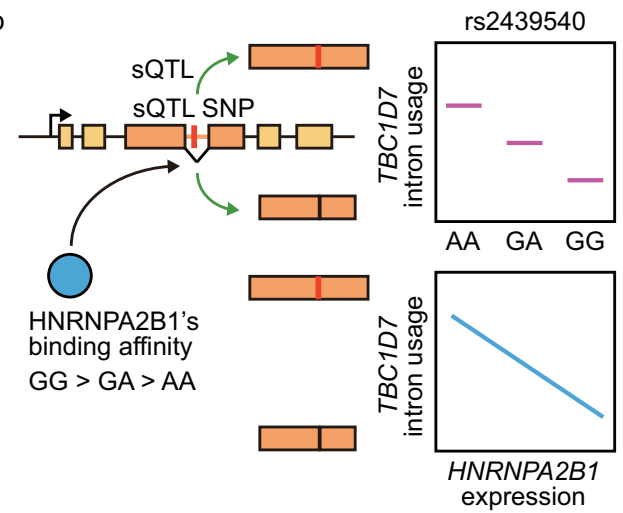

Fig. 3 Genetic backgrounds that affect gene expression and alternative splicing in an age-dependent manner. a. An example of expression quantitative trait loci (eQTLs) during brain aging. Genotypes of eQTL single-nucleotide polymorphisms (SNPs) are associated with the expression of target genes (eGenes) on the same chromosome (cis-eQTLs) or on different chromosomes (trans-eQTLs). Individuals with the GG allele in the SNP rs55675298 exhibit an agedependent increase in the expression of the tumor protein-coding p53 family gene TP73, whereas individuals with the GT or TT allele do not. $\mathbf{b}$. An example of a splicing quantitative trait locus ( $\mathrm{QQTL}$ ) in aged brains. Genotypes of sQTL SNPs are associated with alternative splicing of target genes. The number of $\mathrm{G}$ alleles in the SNP rs 2439540 correlates positively with hnRNP splicing factors, including HNRNPA2B1, and correlates negatively with the intron usage level of TBC1 domain family member 7 (TBC1D7). Consistent with these observations, the mRNA level of HNRNPA2B1 correlates negatively with the intron usage level of TBC1D7.

substantia nigra, and piriform cortex, which are vulnerable to neurodegeneration ${ }^{51}$. In addition, the number of neuroprotective apolipoprotein $\mathrm{E}(A P O E)$ gene $\varepsilon 2$ haplotypes but not $A P O E \varepsilon 4$ haplotypes is associated with reduced expression levels of microglia-enriched genes in aged brains ${ }^{26}$. This pattern suggests that the APOE $\varepsilon 2$ haplotypes moderate the excessive microglial activation that occurs during aging. Overall, these studies show the interaction between the genetic background and agedependent gene expression changes, which may in turn affect human brain aging and age-associated diseases.

\section{Analysis of alternative splicing variants suggests the role of RNA-binding proteins in brain aging}

In addition to gene expression changes, two notable papers that analyzed splicing changes have indicated that human brain aging is associated with alternative splicing $^{17,52}$. Variations in alternative splicing are predominant during prenatal development ${ }^{17}$. After birth, $0.9 \%$ of the genes expressed in the neocortex exhibit differential splicing during postnatal development, whereas $1.4 \%$ of them do so during adulthood ${ }^{17}$. As the brain exhibits the highest level of splicing and preferential expression of genes encoding RNA-binding proteins among all organs of the body ${ }^{16}$, the $1.4 \%$ rate of agedependent splicing changes is substantial compared with the $0.9 \%$ rate.

Remarkably, age-dependent splicing changes do not correlate with changes in the expression of the corresponding genes ${ }^{52}$. The splicing changes that occur during postnatal development and during subsequent aging processes display opposite trends; ${ }^{52} 75 \%$ of segments (exons or introns) show decreased inclusion in transcripts during postnatal development, whereas $77 \%$ of segments show increased inclusion during aging. The majority of segments that follow this "down-up" inclusion pattern are retained introns ${ }^{52}$. Transcripts with retained introns can be targets for nonsense-mediated decay (NMD), which is a crucial system for RNA quality control that degrades various mRNAs, including those with premature stop codons, and regulates alternative splicing ${ }^{53}$. Among the 15 of the 21 genes encoding NMD pathway components that are expressed in the brain, five [up-frameshift suppressor 3 homolog B (UPF3b), suppressor with morphological effect on genitalia homolog 1 (SMG1), SMG5, SMG6, and cancer susceptibility candidate 3 (CASC3)] follow the "down-up" pattern ${ }^{52}$. These findings suggest that components acting in the NMD pathway display characteristic age-dependent expression changes. NMD plays crucial roles in organismal longevity in invertebrates ${ }^{54,55}$. Thus, it will be interesting to evaluate whether the age-dependent expression changes in NMD pathway componentencoding genes contribute to brain aging or ageassociated neurodegenerative diseases.

The expression levels of six genes encoding splicing factors, i.e., polypyrimidine tract-binding proteins 1 and 2 (PTBP1 and PTBP2) and heterogeneous nuclear ribonucleoproteins A1, H1, H3, and F (hnRNPA1, hnRNPH1, $h n R N P H 3$, and $h n R N P F)$, follow the age-dependent "down-up" expression pattern ${ }^{52}$. The expression levels of these genes correlate positively with the "down-up" splicing pattern of particular genes and correlate negatively with the "up-up" or "up-down" splicing patterns of other genes. These relationships may reflect the idea that PTBP1 and PTBP2, which are located in the vicinity of spliced segments ${ }^{56}$, positively or negatively affect segment inclusion $^{57}$. Indeed, a large proportion of PTB targets are segments that exhibit age-related splicing changes ${ }^{35}$.

Analysis of splicing QTLs (sQTLs), also called splicingisoform QTLs (isoQTLs), identified 9,044 genomic loci 
associated with splicing events in 3,006 genes in the aged prefrontal cortex, including the SNP rs $2439540^{58}$ (Fig. $3 \mathrm{~b})$. Although most of these sQTLs do not appear to affect gene expression, 42 loci correlate with the expression and splicing of the genes that contain the loci ${ }^{58}$. In addition, the 9,044 age-associated sQTLs are enriched with potential binding of 18 RNA-binding proteins (RBPs); ${ }^{58}$ PTBP1 is the most prevalently bound RBP, followed by HNRNPC, cleavage and polyadenylation specific factor 7 (CPSF7), and ELAVL1 [embryonic lethal, abnormal vision (ELAV)-like RNA-binding protein 1]. The age-associated sQTLs are also enriched with other hnRNPs ${ }^{58}$. For example, the frequency of the $G$ allele of the SNP rs2439540, which correlates negatively with intron usage in the TBC1 domain family member 7 (TBC1D7) gene, correlates positively with binding of HNRNPA2B1 (Fig. 3b). Moreover, the mRNA levels of hnRNP splicing factors, including HNRNPA2B1 and HNRNPC, correlate negatively with the intron usage levels of hundreds of genes (Fig. 3b), some of which are associated with Alzheimer's disease ${ }^{58}$. These findings suggest that these RBPs, as well as PTBP1 and PTBP2, play roles in the agedependent changes in splicing.

\section{The increased variability of gene expression indicates impairment of genome maintenance and repair with age}

As described above, the transcriptome of the aged brain after the sixth and seventh decades of human life becomes similar to that of the fetal brain ${ }^{18,19}$. However, these changes are not consistently detected between cells or tissues, because the gene expression profiles of brains become heterogeneous during aging ${ }^{5,27}$. This increased variability of gene expression appears to be caused by accumulation of cellular damage and somatic mutations rather than by differences in germline mutations or aging rates between individuals ${ }^{2,59-61}$. Indeed, a pioneering paper that analyzed microarray data obtained from humans of various ages reported that DNA damage is highly enriched in the promoters of genes that are downregulated in aged brains ${ }^{5}$. This finding is consistent with reports showing that gene expression variability is more pronounced among age-dependently downregulated genes than age-dependently upregulated genes ${ }^{33,60}$. The expression of many age-dependently downregulated genes correlates with increased DNA damage ${ }^{5}$, indicating accelerated genome instability during brain aging. The susceptibility of specific brain regions, such as the hippocampus, substantia nigra, and basal ganglia, to DNA damage may be involved in the increased vulnerability of these brain regions to aging compared with that of other regions ${ }^{62}$. Prolonged accumulation of cellular defects often results in immune activation, which causes additional damage ${ }^{63}$, consistent with the increased immune responses observed in aged brains. In summary, agedependent downregulation of genes associated with increased genome instability may underlie the accumulation of stochastic events in aged brain cells. Old cells subsequently become heterogeneous and inflammatory, and these changes may eventually lead to gradual declines in the physiological integrity of the brain.

\section{Noncoding RNAs that participate in human brain aging}

Transcriptomic studies have identified many novel classes of noncoding RNAs (ncRNAs) that are crucial for brain physiology ${ }^{64,65}$. Moreover, functional analyses have established ncRNAs as key regulators of brain aging $^{31,66,67}$. In this section, we highlight the major classes of ncRNAs, i.e., microRNAs (miRNAs), long noncoding RNAs (lncRNAs), and circular RNAs (circRNAs), that participate in human brain aging (Fig. 4).

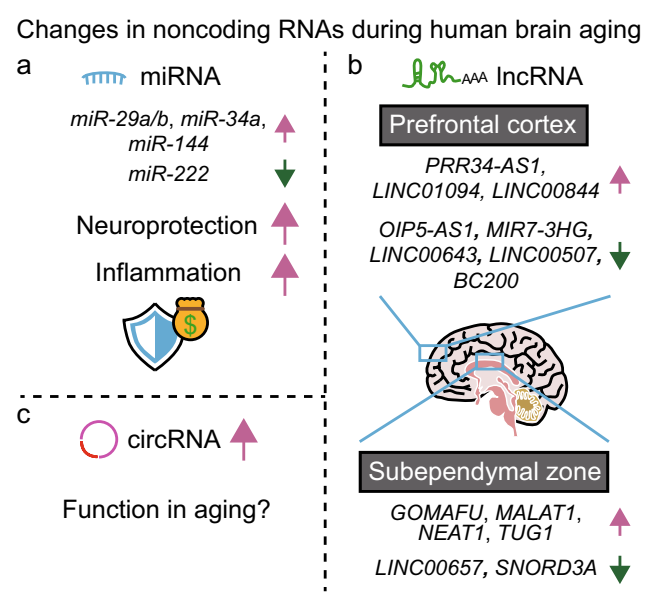

Fig. 4 Noncoding RNAs that participate in human brain aging. a Several microRNAs (miRNAs) enhance neuroprotection in the elderly at the cost of inflammation. The expression of miR-29a/b, miR-34a, and miR-144 increases with age, whereas that of miR-222 decreases. $\mathbf{b}$ Long noncoding RNAs (IncRNAs) display age-dependent expression changes with preserved regional specificity. In the prefrontal cortex, the expression levels of the antisense RNA of the gene encoding proline-rich protein (PRR34-AS1), LINC01094, and LINC00844 correlate positively with age. In contrast, the expression levels of the antisense RNA of the gene encoding opacity-associated (Opa) interacting protein 1 (OIP5-AS1), MIR7-3 host gene (MIR7-3HG), LINC00643, LINC00507, and brain cytoplasmic 200 (BC200) correlate negatively with age. In the subependymal zone, GOMAFU, metastasis-associated lung adenocarcinoma transcript 1 (MALAT1), nuclear paraspeckle assembly transcript 1 (NEAT1), and taurine-upregulated gene 1 (TUG1) display age-dependent upregulation, whereas the expression of LINC00657 and small nucleolar RNA, C/D box 3A (SNORD3A) is downregulated with age. c Circular RNAs (circRNAs) tend to accumulate during brain aging, but the function of their accumulation in aging remains unclear. 


\section{Several miRNAs enhance adaptive neuroprotection in the elderly at the cost of microglial inflammation}

Two independent studies that reported in-depth analysis of miRNAs indicated that expression changes in miRNAs are prominent during human brain aging ${ }^{19,68}$. Overall, the expression of miRNAs in human brains decreases with age after adolescence; in contrast, a subset of miRNAs display age-dependent upregulation ${ }^{19,68}$. In some cases, age-dependent expression changes in miRNAs appear to be protective against neurodegenerative diseases and brain aging. The expression of miR-144 increases with age in the cerebellum and suppresses the expression of the ataxin 1 (ATXN1) gene ${ }^{69}$ (Fig. 4a). Because trinucleotide repeat expansion mutations in $A T X N 1$, which augment the glutamine repeats in the encoded protein, underlie the development of spinocerebellar ataxia $1(\mathrm{SCA} 1)^{70}$, miR-144 may play a protective role against this neurodegenerative disease. In addition, an age-dependent decrease in $m i R-222$ expression appears to result in derepression of its target genes ${ }^{19}$, including REV1/deoxycytidyl transferase, which regulates the DNA damage response ${ }^{71}$ (Fig. 4a). This process may eventually reduce the excessive DNA damage in aged brains.

However, the adaptive neuroprotection afforded by agedependent expression changes in several miRNAs also seems to be associated with health complications, including excessive microglial inflammation ${ }^{31}$. For example, miR-34a, which acts as a tumor suppressor in neuroblastoma ${ }^{72}$, exhibits an age-dependent increase, which in turn downregulates neuronal genes, thus possibly participating in brain aging ${ }^{19}$ (Fig. 4a). In addition, the expression of $m i R-29 a / b$ is dramatically increased with age in the superior frontal gyrus and the postcentral parietal area ${ }^{19,73}$ and exerts neuroprotective functions $^{74,75}$, as well as induces excessive microglial inflammation $^{73}$ (Fig. 4a). Low expression of $m i R-29 a / b$ is observed in the brains of patients with sporadic Alzheimer's disease and correlates with high BACE1/ $\beta$-secretase levels $^{74}$ and a high amyloid beta load ${ }^{76}$, again suggesting the neuroprotective role of miR-29a/b. Conversely, miR$29 a / b$ expression correlates negatively with the mRNA levels of insulin-like growth factor 1 (IGF1) and $C X_{3} C R 1^{73}$, both of which inhibit excessive microglial activation $^{42,43,77}$. Thus, the age-dependent increases in miR-29a/b levels seem to protect against neurodegeneration in the elderly at the cost of excessive microglial inflammation (Fig. 4a).

\section{IncRNAs display high regional specificity in aging human brains}

Multiple studies focusing on analysis of lncRNAs have reported that aged human brains exhibit expression changes in various lncRNAs with regional specificity ${ }^{78-81}$
(Fig. 4b). Among the 383 lncRNAs that are differentially expressed between young and aged samples from four brain regions, only eight exhibit consistent changes in all brain regions ${ }^{80}$, indicating the high regional specificity of lncRNAs.

In the prefrontal cortex, the expression levels of the antisense RNA of the gene encoding proline-rich protein (PRR34-AS1), LINC01094, and LINC00844 correlate positively with age, whereas the expression levels of the antisense RNA of the gene encoding opacity-associated $(\mathrm{Opa})$ interacting protein 1 (OIP5-AS1), MIR7-3 host gene (MIR7-3HG), LINC00643, LINC00507, and brain cytoplasmic $200(B C 200)$ correlate negatively with age $\mathrm{e}^{78,81}$ (Fig. 4b). These age-dependent changes in the expression of IncRNAs in the prefrontal cortex reflect cognitive declines with age. LINC00507, which has ribosomebinding activity and micropeptide-coding potential, is specific to cortical regions in primates ${ }^{82}$, suggesting that its downregulation with age is associated with cognitive decline. Another lncRNA, BC200, inhibits local mRNA translation in dendrites and synapses ${ }^{83,84}$. The expression level of $B C 200$ in the prefrontal cortex decreases by $65 \%$ between the ages of 49 and 86 years $^{78}$ and correlates with age-dependent declines in synaptic functions ${ }^{85}$.

In the subependymal zone of the adult brain, which contains neural stem cells ${ }^{86}$, GOMAFU [also called myocardial infarction associated transcript (MIAT) and retinal noncoding RNA 2 (RNCR2)], metastasis-associated lung adenocarcinoma transcript 1 (MALAT1), nuclear paraspeckle assembly transcript 1 (NEAT1), and taurineupregulated gene 1 (TUG1) exhibit age-dependent upregulation, whereas the expression of LINC00657 and small nucleolar RNA, C/D box 3A (SNORD3A) is downregulated with age ${ }^{79}$ (Fig. 4b). GOMAFU acts as a scaffold for the sequestration of splicing factors and modulates the splicing of transcripts that are required for synaptic plasticity ${ }^{87}$. Neuronal activation decreases the expression of GOMAFU and releases splicing factors into the nucleoplasm $^{87}$. In addition to GOMAFU, MALAT1 and NEAT1 are located in nuclear speckles and paraspeckles, respectively, where they regulate alternative splicing in neurons by sequestering splicing factors ${ }^{88}$. However, the mechanisms by which MALAT1 and NEAT1 participate in brain aging remain poorly understood. TUG1 sequesters miR-9, and its upregulation leads to release of BCL2 like 11 (BCL2L11) mRNA from miR-9 to promote apoptosis in rats with ischemia ${ }^{89}$. Thus, the agedependent increase in the expression of TUG1 in the subependymal zone may indicate the acceleration of neuronal apoptosis in aged brains ${ }^{79}$.

The age-dependent expression changes in a subset of lncRNAs also display specificity for other brain regions. The postcentral gyrus and superior frontal gyrus exhibit a larger number of differentially expressed lncRNAs than 
does the entorhinal cortex or hippocampus ${ }^{80}$. The majority of the age-dependent changes in lncRNA expression manifest as upregulation in all regions except the entorhinal cortex ${ }^{80}$. Overall, lncRNAs appear to be more region-specific than mRNAs in aging human brains.

\section{circRNA levels tend to increase during human brain aging}

circRNAs are mostly noncoding RNAs that are formed by backsplicing events; ${ }^{90}$ however, the biological functions of circRNAs remain largely unexplored. Unlike linear RNAs, circRNAs are covalently linked at their termini and lack $3^{\prime}$ poly(A) tails; therefore, circRNAs display high resistance against exonucleases ${ }^{91-93}$. This property endows circRNAs with enhanced stability and raises the possibility that the levels of circRNAs remain high in nonproliferating cells. The levels of circRNAs do not correlate positively with those of their cognate mRNA isoforms ${ }^{94,95}$. circRNAs are highly expressed in brain tissues compared with nonbrain tissues in mice and humans ${ }^{94}$. Importantly, circRNAs display agedependent accumulation in the brains of mice and a nonhuman primate, the rhesus macaque ${ }^{95,96}$ (Fig. 4c). In addition, certain circRNAs are specifically detected in patients with Alzheimer's disease ${ }^{97}$. Therefore, although circRNAs have not been directly characterized in the aging human brain, it seems likely that the levels of circRNAs increase during human brain aging. It will be important to determine by future research whether the accumulation of circRNAs plays a role in aging and agerelated diseases.

\section{Conclusions and perspectives}

Transcriptome analysis of human brain aging offers a comprehensive view of neurocognitive aging and neurodegenerative diseases. Here, we reviewed studies regarding the transcriptome changes associated with human brain aging. Decreased synaptic function and increased immune function are prevalent in most brain regions during aging, but other age-associated transcriptome changes display cellular or regional specificities. Gene expression in glial cells, including astrocytes and oligodendrocytes, is dramatically altered during brain aging and is accompanied by loss of the regional identities of these genes. Age-dependent gene expression changes are also affected by genetic backgrounds and splicing events. The role of RBPs, including splicing factors, is important for the splicing events in many transcripts during brain aging. Increased intersample heterogeneity is a property that implies the impairment of genome maintenance and repair in aged brains. The role of age-associated expression changes in noncoding RNAs, including miRNAs, lncRNAs, and circRNAs, remains less well understood than those in mRNAs. Thus, future studies of these noncoding RNAs will lead to a deeper understanding of brain aging at the molecular level.

The emergence of single-cell RNA-seq technology has enhanced the potential of transcriptome analysis and will help improve the understanding of human brain aging at the single-cell level. Specifically, investigation of singlecell transcriptomes provides unbiased insights into the subtypes and the diversities of brain cells ${ }^{98,99}$. Single-cell RNA-seq overcomes the problem of bulk RNA-seq, i.e., that the transcriptional contribution from minor cell types is masked by major cell types in tissues. Currently, examination of single nuclei is usually preferred ${ }^{98}$. However, this method cannot estimate the expression of cytosolic transcripts. Therefore, optimization of singlecell isolation is required for brain transcriptome analysis at the single-cell level. A recent study reported successful analysis of the single-cell transcriptome in the aging mouse brain by introducing sophisticated brain tissue dissociation steps ${ }^{100}$. Thus, future research employing single-cell RNA-seq technology with human brain samples will pave the way for providing new insights into human brain aging. In addition, by combining this singlecell technique with in situ hybridization ${ }^{101}$, transcriptome analysis will allow spatial separation for visualizing gene expression in human brain cells during aging.

One of the limitations of previous research on transcriptome analysis of the human brain is that the majority of participants in postmortem brain studies are of Caucasian ethnicity. Among 1007 individuals with specified ethnicity in previous studies (Table 1), 733 (72.8\%) and 32 (3.2\%) were Caucasian and European, respectively. African-American (198, 19.7\%) and African (16, 1.6\%), Hispanic $(18,1.8 \%)$ and Asian $(8,0.8 \%)$ populations comprised a minor fraction. Thus, our understanding of human brain aging may be effective for only a subset of the human population at best. Future investigation of transcriptomes obtained from a wide spectrum of ethnic groups will be crucial for a comprehensive understanding of human brain aging.

In conclusion, understanding the transcriptome in aged human brains will lead to the early detection of cognitive aging and the development of interventions that delay brain aging at the molecular level. Furthermore, given the close relationship between brain aging and neurodegenerative diseases, the establishment of transcriptome-based hallmarks of human brain aging will facilitate the diagnosis and treatment of these disorders.

\footnotetext{
Acknowledgements

We thank all Lee laboratory members for help and discussion. This study is supported by the Korean Government (MSICT) through National Research Foundation of Korea (NRF) grants NRF-2019R1A3B2067745 to S-J.V.L. and NRF2019R1A6A3A01093131 to S.H.
}

Conflict of interest

The authors declare that they have no conflict of interest. 


\section{Publisher's note}

Springer Nature remains neutral with regard to jurisdictional claims in published maps and institutional affiliations.

\section{Received: 8 May 2020 Revised: 15 September 2020 Accepted: 22} September 2020.

Published online: 26 November 2020

\section{References}

1. Morrison, J. H. \& Baxter, M. G. The ageing cortical synapse: hallmarks and implications for cognitive decline. Nat. Rev. Neurosci. 13, 240-250 (2012).

2. Lopez-Otin, C., Blasco, M. A., Partridge, L., Serrano, M. \& Kroemer, G. The hallmarks of aging. Cell 153, 1194-1217 (2013).

3. Kennedy, B. K. et al. Geroscience: linking aging to chronic disease. Cell 159 709-713 (2014).

4. Pakkenberg, B. et al. Aging and the human neocortex. Exp. Gerontol. 38, 95-99 (2003).

5. Lu, T. et al. Gene regulation and DNA damage in the ageing human brain Nature 429, 883-891 (2004).

6. Berchtold, N. C. et al. Gene expression changes in the course of normal brain aging are sexually dimorphic. Proc. Natl Acad. Sci. USA 105, 15605-15610 (2008).

7. Loerch, P. M. et al. Evolution of the aging brain transcriptome and synaptic regulation. PLOS ONE 3, e3329 (2008).

8. Khan, S. S., Singer, B. D. \& Vaughan, D. E. Molecular and physiological manifestations and measurement of aging in humans. Aging Cell 16, 624-633 (2017).

9. Velculescu, V. E. et al. Characterization of the yeast transcriptome. Cell 88 , 243-251 (1997).

10. Pietu, $G$. et al. The Genexpress IMAGE knowledge base of the human brain transcriptome: a prototype integrated resource for functional and computational genomics. Genome Res. 9, 195-209 (1999).

11. Lowe, R., Shirley, N., Bleackley, M., Dolan, S. \& Shafee, T. Transcriptomics technologies. PloS Comput Biol. 13, e1005457 (2017).

12. Wang, Z., Gerstein, M. \& Snyder, M. RNA-Seq: a revolutionary tool for transcriptomics. Nat. Rev. Genet. 10, 57-63 (2009).

13. Wolf, J. B. Principles of transcriptome analysis and gene expression quantification: an RNA-seq tutorial. Mol. Ecol. Resour. 13, 559-572 (2013).

14. Hawrylycz, M. J. et al. An anatomically comprehensive atlas of the adult human brain transcriptome. Nature 489, 391-399 (2012).

15. Hawrylycz, M. et al. Canonical genetic signatures of the adult human brain Nat. Neurosci. 18, 1832-1844 (2015).

16. Mele, $M$. et al. The human transcriptome across tissues and individuals. Science 348, 660-665 (2015).

17. Kang, H. J. et al. Spatio-temporal transcriptome of the human brain. Nature 478, 483-489 (2011)

18. Colantuoni, $C$. et al. Temporal dynamics and genetic control of transcription in the human prefrontal cortex. Nature 478, 519-523 (2011).

19. Somel, M. et al. MicroRNA, mRNA, and protein expression link development and aging in human and macaque brain. Genome Res. 20 1207-1218 (2010)

20. Soreq, L. et al. Major shifts in glial regional identity are a transcriptional hallmark of human brain aging. Cell Rep. 18, 557-570 (2017).

21. Erraji-Benchekroun, L. et al. Molecular aging in human prefrontal cortex is selective and continuous throughout adult life. Biol. Psychiatry 57, 549-558 (2005).

22. Cribbs, D. H. et al. Extensive innate immune gene activation accompanies brain aging, increasing vulnerability to cognitive decline and neurodegeneration: a microarray study. J. Neuroinflammation 9, 179 (2012).

23. Norden, D. M. \& Godbout, J. P. Review: microglia of the aged brain: primed to be activated and resistant to regulation. Neuropathol. Appl. Neurobiol. 39 19-34 (2013).

24. Primiani, C. T. et al. Coordinated gene expression of neuroinflammatory and cell signaling markers in dorsolateral prefrontal cortex during human brain development and aging. PLOS ONE 9, e110972 (2014).

25. Zullo, J. M. et al. Regulation of lifespan by neural excitation and REST. Nature 574, 359-364 (2019)

26. Olah, M. et al. A transcriptomic atlas of aged human microglia. Nat. Commun. 9, 539 (2018).
27. Naumova, O. Y. et al. Age-related changes of gene expression in the neocortex: preliminary data on RNA-Seq of the transcriptome in three functionally distinct cortical areas. Dev. Psychopathol. 24, 1427-1442 (2012).

28. Berchtold, N. C. et al. Synaptic genes are extensively downregulated across multiple brain regions in normal human aging and Alzheimer's disease. Neurobiol. Aging 34, 1653-1661 (2013).

29. Riascos, D. et al. Alterations of Ca2+-responsive proteins within cholinergic neurons in aging and Alzheimer's disease. Neurobiol. Aging 35, 1325-1333 (2014)

30. Rice, R. A., Berchtold, N. C., Cotman, C. W. \& Green, K. N. Age-related downregulation of the CaV3.1 T-type calcium channel as a mediator of amyloid beta production. Neurobiol. Aging 35, 1002-1011 (2014).

31. Mohan, A., Mather, K. A., Thalamuthu, A., Baune, B. T. \& Sachdev, P. S. Gene expression in the aging human brain: an overview. Curr. Opin. Psychiatry 29, 159-167 (2016).

32. Brinkmeyer-Langford, C. L., Guan, J., Ji, G. \& Cai, J. J. Aging shapes the population-mean and -dispersion of gene expression in human brains. Front. Aging Neurosci. 8, 183 (2016).

33. Donertas, H. M. et al. Gene expression reversal toward pre-adult levels in the aging human brain and age-related loss of cellular identity. Sci. Rep. 7, 5894 (2017).

34. Lu, T. et al. REST and stress resistance in ageing and Alzheimer's disease. Nature 507, 448-454 (2014)

35. Tollervey, J. R. et al. Analysis of alternative splicing associated with aging and neurodegeneration in the human brain. Genome Res. 21, 1572-1582 (2011).

36. Li, Q. \& Barres, B. A. Microglia and macrophages in brain homeostasis and disease. Nat. Rev. Immunol. 18, 225-242 (2018).

37. Kuhn, S., Gritti, L., Crooks, D. \& Dombrowski, Y. Oligodendrocytes in development, myelin generation and beyond. Cells 8, 1424 (2019).

38. Mitra, K., Carvunis, A. R., Ramesh, S. K. \& Ideker, T. Integrative approaches for finding modular structure in biological networks. Nat. Rev. Genet. 14, 719-732 (2013)

39. Bae, S. H. et al. Decipher reliable biomarkers of brain aging by integrating literature-based evidence with interactome data. Exp. Mol. Med. 50, 28 (2018).

40. Dillman, A. A. et al. Transcriptomic profiling of the human brain reveals that altered synaptic gene expression is associated with chronological aging. Sci. Rep. 7, 16890 (2017)

41. Wehrspaun, C. C., Haerty, W. \& Ponting, C. P. Microglia recapitulate a hematopoietic master regulator network in the aging human frontal cortex. Neurobiol. Aging 36, 2443 e9-2443. e20 (2015).

42. Cardona, A. E. et al. Control of microglial neurotoxicity by the fractalkine receptor. Nat. Neurosci. 9, 917-924 (2006).

43. Bachstetter, A. D. et al. Fractalkine and CX3CR1 regulate hippocampal neurogenesis in adult and aged rats. Neurobiol. Aging 32, 2030-2044 (2011).

44. Morley, M. et al. Genetic analysis of genome-wide variation in human gene expression. Nature 430, 743-747 (2004).

45. Grundberg, E. et al. Mapping cis- and trans-regulatory effects across multiple tissues in twins. Nat. Genet. 44, 1084-1089 (2012)

46. Yang, A. et al. p73-deficient mice have neurological, pheromonal and inflammatory defects but lack spontaneous tumours. Nature 404, 99-103 (2000).

47. Pozniak, C. D. et al. An anti-apoptotic role for the p53 family member, p73, during developmental neuron death. Science 289, 304-306 (2000).

48. Pozniak, C. D. et al. p73 is required for survival and maintenance of CNS neurons. J. Neurosci. 22, 9800-9809 (2002).

49. Li, Q. et al. TP73 allelic expression in human brain and allele frequencies in Alzheimer's disease. BMC Med. Genet. 5, 14 (2004).

50. Wetzel, M. K. et al. p73 regulates neurodegeneration and phospho-tau accumulation during aging and Alzheimer's disease. Neuron 59, 708-721 (2008).

51. Stephan, A. H. et al. A dramatic increase of C1q protein in the CNS during normal aging. J. Neurosci. 33, 13460-13474 (2013).

52. Mazin, P. et al. Widespread splicing changes in human brain development and aging. Mol. Syst. Biol. 9, 633 (2013).

53. Kim, Y. K. \& Maquat, L. E. UPFront and center in RNA decay: UPF1 in nonsense-mediated mRNA decay and beyond. RNA 25, 407-422 (2019).

54. Son, H. G. et al. RNA surveillance via nonsense-mediated mRNA decay is crucial for longevity in daf-2/insulin/IGF-1 mutant C. elegans. Nat. Commun. 8, 14749 (2017).

55. Tabrez, S. S., Sharma, R. D., Jain, V., Siddiqui, A. A. \& Mukhopadhyay, A. Differential alternative splicing coupled to nonsense-mediated decay of 
mRNA ensures dietary restriction-induced longevity. Nat. Commun. 8, 306 (2017).

56. Gooding, C., Roberts, G. C. \& Smith, C. W. Role of an inhibitory pyrimidine element and polypyrimidine tract binding protein in repression of a regulated alpha-tropomyosin exon. RNA 4, 85-100 (1998).

57. Llorian, M. et al. Position-dependent alternative splicing activity revealed by global profiling of alternative splicing events regulated by PTB. Nat. Struct. Mol. Biol. 17, 1114-1123 (2010).

58. Raj, T. et al. Integrative transcriptome analyses of the aging brain implicate altered splicing in Alzheimer's disease susceptibility. Nat. Genet. 50, 1584-1592 (2018).

59. Kirkwood, T. B. Understanding the odd science of aging. Cell 120, 437-447 (2005)

60. Somel, M., Khaitovich, P., Bahn, S., Paabo, S. \& Lachmann, M. Gene expression becomes heterogeneous with age. Curr. Biol. 16, R359-R360 (2006).

61. Kowald, A. \& Kirkwood, T. B. L. Can aging be programmed? A critical literature review. Aging Cell 15, 986-998 (2016).

62. Fu, H., Hardy, J. \& Duff, K. E. Selective vulnerability in neurodegenerative diseases. Nat. Neurosci. 21, 1350-1358 (2018).

63. Ioannidou, A., Goulielmaki, E. \& Garinis, G. A. DNA damage: from chronic inflammation to age-related deterioration. Front. Genet. 7, 187 (2016).

64. Qureshi, I. A. \& Mehler, M. F. Emerging roles of non-coding RNAs in brain evolution, development, plasticity and disease. Nat. Rev. Neurosci. 13, 528-541 (2012).

65. Salta, E. \& De Strooper, B. Noncoding RNAs in neurodegeneration. Nat. Rev. Neurosci. 18, 627-640 (2017).

66. Earls, L. R., Westmoreland, J. J. \& Zakharenko, S. S. Non-coding RNA regulation of synaptic plasticity and memory: implications for aging. Ageing Res Rev. 17, 34-42 (2014).

67. Abraham, K. J., Ostrowski, L. A. \& Mekhail, K. Non-coding RNA molecules connect calorie restriction and lifespan. J. Mol. Biol. 429, 3196-3214 (2017).

68. Beveridge, N. J. et al. Maturation of the human dorsolateral prefrontal cortex coincides with a dynamic shift in microRNA expression. Schizophr. Bull. $\mathbf{4 0}$ 399-409 (2014).

69. Persengiev, S., Kondova, I., Otting, N., Koeppen, A. H. \& Bontrop, R. E Genome-wide analysis of miRNA expression reveals a potential role for miR144 in brain aging and spinocerebellar ataxia pathogenesis. Neurobiol. Aging 32, 2316 e17-2316 e27 (2011).

70. Orr, H. T. et al. Expansion of an unstable trinucleotide CAG repeat in spinocerebellar ataxia type 1. Nat. Genet. 4, 221-226 (1993).

71. Lin, W. et al. The human REV1 gene codes for a DNA template-dependent dCMP transferase. Nucleic Acids Res. 27, 4468-4475 (1999).

72. Cole, K. A. et al. A functional screen identifies miR-34a as a candidate neuroblastoma tumor suppressor gene. Mol. Cancer Res. 6, 735-742 (2008).

73. Fenn, A. M. et al. Increased micro-RNA 29b in the aged brain correlates with the reduction of insulin-like growth factor-1 and fractalkine ligand. Neurobiol. Aging 34, 2748-2758 (2013).

74. Hebert, S. S. et al. Loss of microRNA cluster miR-29a/b-1 in sporadic Alzheimer's disease correlates with increased BACE1/beta-secretase expression. Proc. Natl Acad. Sci. USA 105, 6415-6420 (2008).

75. Ouyang, Y. B. et al. Astrocyte-enriched miR-29a targets PUMA and reduces neuronal vulnerability to forebrain ischemia. Glia 61, 1784-1794 (2013).

76. Li, R. et al. Amyloid beta peptide load is correlated with increased betasecretase activity in sporadic Alzheimer's disease patients. Proc. Natl Acad. Sci. USA 101, 3632-3637 (2004).

77. O'Connor, J. C. et al. Regulation of IGF-I function by proinflammatory cytokines: at the interface of immunology and endocrinology. Cell Immunol. 252 91-110 (2008).

78. Mus, E., Hof, P. R. \& Tiedge, H. Dendritic BC200 RNA in aging and in Alzheimer's disease. Proc. Natl Acad. Sci. USA 104, 10679-10684 (2007).
79. Barry, G., Guennewig, B., Fung, S., Kaczorowski, D. \& Weickert, C. S. Long noncoding ma expression during aging in the human subependymal zone. Front. Neurol. 6, 45 (2015)

80. Zhou, M., Zhao, H., Wang, X., Sun, J. \& Su, J. Analysis of long noncoding RNAs highlights region-specific altered expression patterns and diagnostic roles in Alzheimer's disease. Brief. Bioinform. 20, 598-608 (2019).

81. Cao, M., Li, H., Zhao, J., Cui, J. \& Hu, G. Identification of age- and genderassociated long noncoding RNAs in the human brain with Alzheimer's disease. Neurobiol. Aging 81, 116-126 (2019).

82. Mills, J. D. et al. LINC00507 is specifically expressed in the primate cortex and has age-dependent expression patterns. J. Mol. Neurosci. 59, 431-439 (2016).

83. Tiedge, H., Chen, W. \& Brosius, J. Primary structure, neural-specific expression, and dendritic location of human BC200 RNA. J. Neurosci. 13, 2382-2390 (1993).

84. Kondrashov, A. V. et al. Inhibitory effect of naked neural BC1 RNA or BC200 RNA on eukaryotic in vitro translation systems is reversed by poly(A)-binding protein (PABP). J. Mol. Biol. 353, 88-103 (2005).

85. Hof, P. R. \& Morrison, J. H. The aging brain: morphomolecular senescence of cortical circuits. Trends Neurosci. 27, 607-613 (2004)

86. Morante-Redolat, J. M. \& Porlan, E. Neural stem cell regulation by adhesion molecules within the subependymal niche. Front. Cell Dev. Biol. 7 102 (2019).

87. Barry, G. et al. The long non-coding RNA Gomafu is acutely regulated in response to neuronal activation and involved in schizophrenia-associated alternative splicing. Mol. Psychiatry 19, 486-494 (2014).

88. Bernard, D. et al. A long nuclear-retained non-coding RNA regulates synaptogenesis by modulating gene expression. EMBO J. 29, 3082-3093 (2010).

89. Chen, S. et al. LncRNA TUG1 sponges microRNA-9 to promote neurons apoptosis by up-regulated Bcl2111 under ischemia. Biochem. Biophys. Res. Commun. 485, 167-173 (2017).

90. Cocquerelle, C., Mascrez, B., Hetuin, D. \& Bailleul, B. Mis-splicing yields circular RNA molecules. FASEB J. 7, 155-160 (1993).

91. Hansen, T. B et al. Natural RNA circles function as efficient microRNA sponges. Nature 495, 384-388 (2013).

92. Memczak, S. et al. Circular RNAs are a large class of animal RNAs with regulatory potency. Nature 495, 333-338 (2013).

93. Li, X., Yang, L. \& Chen, L. L. The biogenesis, functions, and challenges of circular RNAs. Mol. Cell 71, 428-442 (2018).

94. Rybak-Wolf, A. et al. Circular RNAs in the mammalian brain are highly abundant, conserved, and dynamically expressed. Mol. Cell 58, 870-885 (2015).

95. Gruner, H., Cortes-Lopez, M., Cooper, D. A., Bauer, M. \& Miura, P. CircRNA accumulation in the aging mouse brain. Sci. Rep. 6, 38907 (2016).

96. $\mathrm{Xu}, \mathrm{K}$. et al. Annotation and functional clustering of circRNA expression in rhesus macaque brain during aging. Cell Discov. 4, 48 (2018).

97. Zhang, Y., Yu, F., Bao, S. \& Sun, J. Systematic characterization of circular RNAassociated CeRNA network identified novel circRNA Biomarkers in Alzheimer's disease. Front. Bioeng. Biotechnol. 7, 222 (2019).

98. Bushman, D. M. et al. Genomic mosaicism with increased amyloid precursor protein (APP) gene copy number in single neurons from sporadic Alzheimer's disease brains. Elife 4, e05116 (2015)

99. Poulin, J. F., Tasic, B., Hjerling-Leffler, J., Trimarchi, J. M. \& Awatramani, R. Disentangling neural cell diversity using single-cell transcriptomics. Nat. Neurosci. 19, 1131-1141 (2016).

100. Ximerakis, M. et al. Single-cell transcriptomic profiling of the aging mouse brain. Nat. Neurosci. 22, 1696-1708 (2019).

101. Stahl, P. L. et al. Visualization and analysis of gene expression in tissue sections by spatial transcriptomics. Science 353, 78-82 (2016) 